\title{
SOLITARY FIBROUS TUMOR ASSOCIATED WITH HYPOGLYCEMIA: AN EXAMPLE OF THE DOEGE- POTTER SYNDROME
}

\author{
Martin H. Chamberlain, FRCS, and David P Taggart, MD, FRCS, Oxford, United Kingdom
}

A previously fit and healthy 48-year-old man was admitted with a 2-week history of transient episodes of loss of consciousness and vagueness associated with sweating and nausea. He was a nonsmoker with an uneventful medical history. The patient also had recently begun having increasing breathlessness on climbing 2 flights of stairs. He had not noticed any change in his weight.

General examination showed no abnormalities except for tracheal deviation to the left with very little air entry into the right lung, which was dull to percussion. No focal neurologic abnormalities were observed.

Results of blood biochemical and hematologic studies were within normal limits except for a random blood glucose value that was $2.6 \mathrm{mmol} / \mathrm{L}$.

Chest radiography showed a large space-occupying lesion in the right hemithorax causing the right lung to collapse and displacing the mediastinum to the left. Chest computed tomographic (CT) scan revealed a large well-circumscribed heterogeneous mass taking up most of the right hemithorax and compressing the adjacent lung with associated mediastinal shift to the left (Fig 1). Pleural relationships were unclear. There was concern that the mediastinal structures might be involved, but no bone, liver, or spleen involvement was noted and no lymphadenopathy was seen. Contrast enhancement suggested areas of necrosis. Two CT-guided biopsies gave the diagnosis of subpleural fibroma, a solitary fibrous tumor. Brain CT scan showed no abnormalities.

At operation, the patient was placed supine with a wedge under his right side. A median sternotomy was performed. This allowed reasonable access to the tumor and would enable cardiopulmonary bypass to be used should it become necessary. To improve access, we used a right anterolateral thoracotomy that extended through the right hemisternum in the 5th intercostal space.

Operative findings confirmed the CT appearances. The tumor was encapsulated and had a posterior pedicle attached to the mediastinal pleura, with numerous adhesions. There was no invasion into the mediastinal structures. The tumor

From the Oxford Heart Centre, The John Radcliffe Hospital, Oxford, United Kingdom.

Received for publication Aug 4, 1999; accepted for publication Aug 17, 1999.

Address for reprints: D. P. Taggart, MD, FRCS, Oxford Heart Centre, The John Radcliffe Hospital, Headington, Oxford, Oxfordshire, England OX3 9DU.

J Thorac Cardiovasc Surg 2000;119:185-7

Copyright $(2000$ by Mosby, Inc.

$0022-5223 / 2000 \$ 12.00+0 \quad \mathbf{1 2 / 5 4 / 1 0 2 2 9 3}$

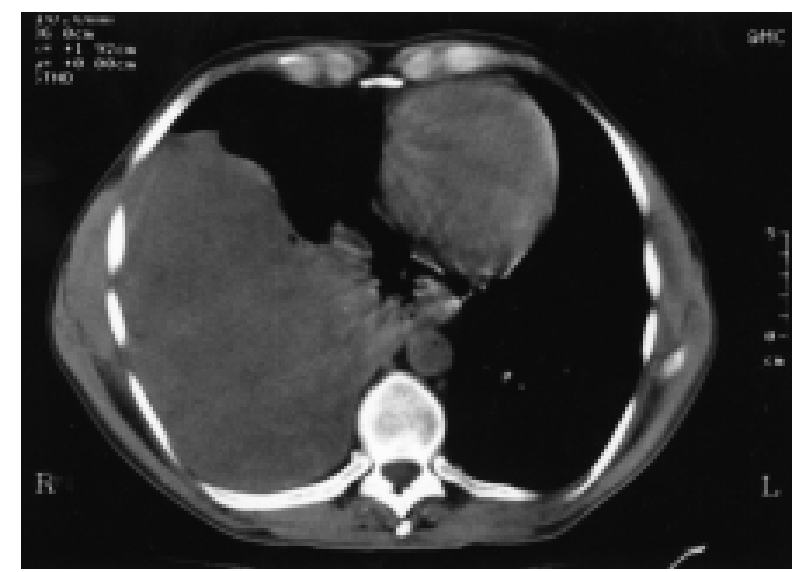

Fig 1. Preoperative CT scan showing the mass in the right hemithorax with associated mediastinal shift to the left.

was dissected free and removed from the thorax en masse. The right lung immediately re-expanded. Two chest drains were inserted.

Postoperatively, the blood sugar measurements rapidly returned to normal limits. The operative incisions healed without complication and the patient was discharged on day 17 .

The tumor was a lobulated, rubbery mass weighing $3070 \mathrm{~g}$ with dimensions of $23 \times 21 \times 12 \mathrm{~cm}$ (Fig 2). It had no distinctive features. Microscopically, it was principally composed of spindle cells in fascicles or in a haphazard arrangement with large areas of hyalinization and degeneration (Fig 3 ). The spindle cells were regular with minimal nuclear pleomorphism, and there was no mitotic activity. A thin rim of connective tissue merging with the underlying cells enclosed the entire tumor. There was no evidence of malignancy. Immunohistochemical stains for CD34 and vimentin (Fig 4) showed strongly positive reactions. These are characteristic of a solitary fibrous tumor.

Discussion. A solitary fibrous tumor is an uncommon spindle cell neoplasm usually associated with serosal surfaces. It was first described in relation to the pleura, ${ }^{1}$ where it is most commonly found, but occurrence in extrapleural sites is increasingly reported. ${ }^{2-4}$ These extrapleural sites are often associated with the upper respiratory tract and adjacent structures. They usually cause symptoms because of compression of neighboring structures.

Within the thoracic cavity the pleura is the most frequent site of solitary fibrous tumors, with $80 \%$ originating from the visceral and $20 \%$ from the parietal pleura. ${ }^{5}$ They can behave like benign $(88 \%)$ or malignant ${ }^{5}$ tumors. They range in size 


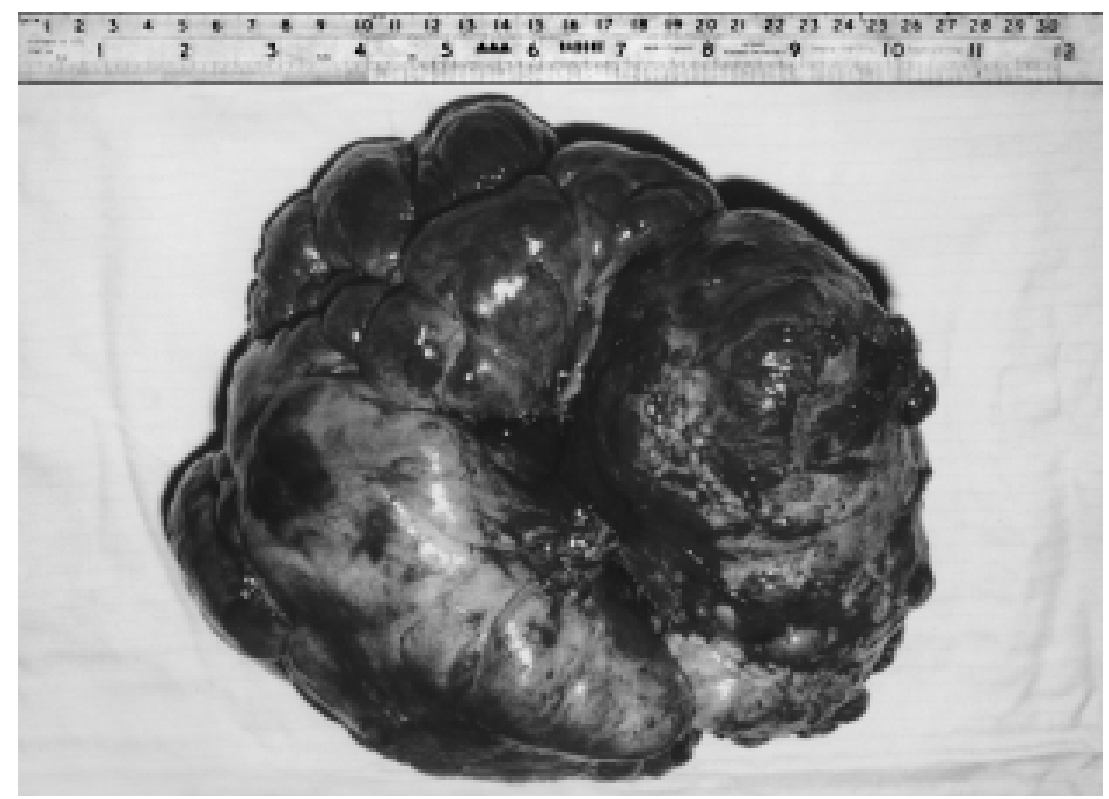

Fig 2. Postoperative photograph of the resected tumor demonstrating its lobulated appearance and extensive size.

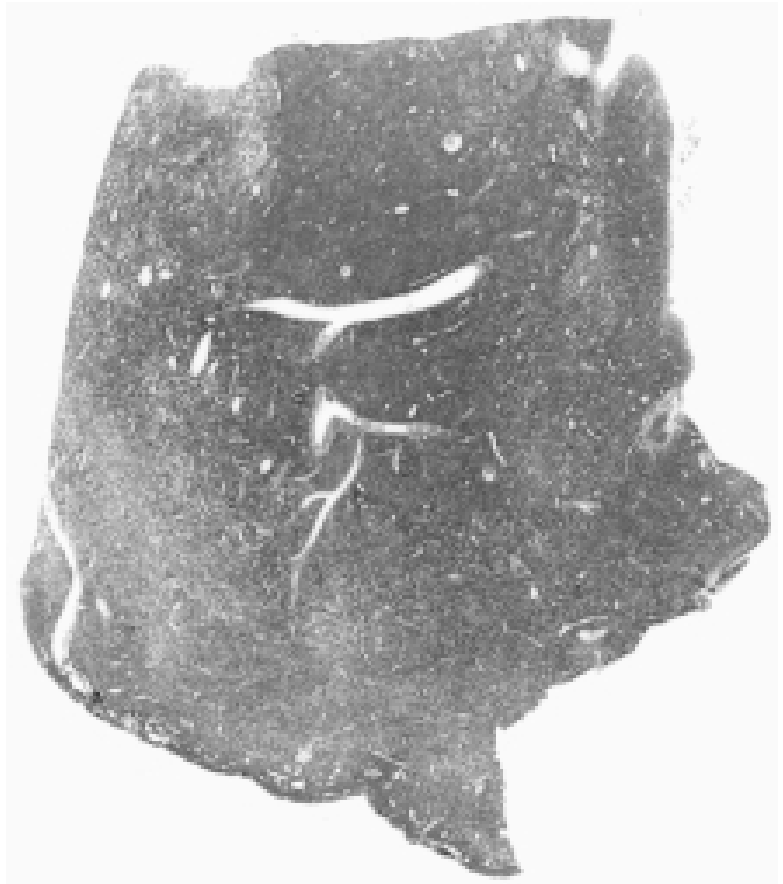

Fig 3. Slide of tumor tissue stained with hematoxylin and eosin.

from 1 to $36 \mathrm{~cm}$ in maximum diameter with a mean of $6 \mathrm{~cm}^{5}$ and are usually well-circumscribed, pedunculated lesions.

Histologically, they are formed of cellular areas of alternating hyalinized and/or necrotic areas. They contain spindleshaped cells that typically have minimal nuclear pleomor-

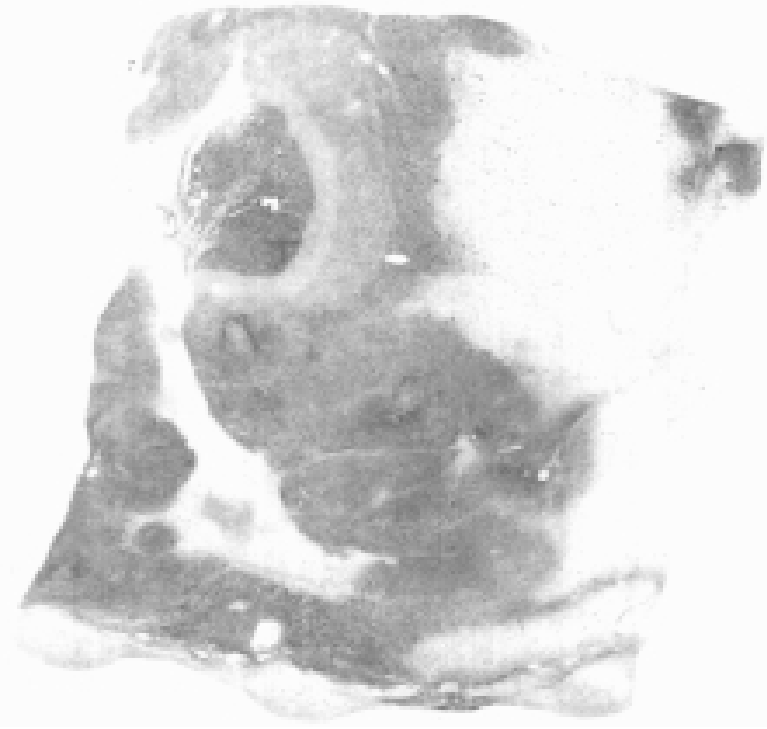

Fig 4. Immunohistochemical staining of the tumor with vimentin.

phism and rare or absent mitotic activity. They are distinguished by positive staining for both CD34 and vimentin. ${ }^{6}$

Solitary fibrous tumors may be incidental findings or may be associated with cough, chest pain, or dyspnea. If hypoglycemia is demonstrated in connection with a solitary fibrous tumor, it is referred to as the Doege-Potter syndrome. Briselli, Mark, and Dickersin ${ }^{5}$ showed that $4 \%$ of solitary fibrous tumors are associated with hypoglycemia and that 
this is more common in large tumors with a high mitotic rate. Insulin-like growth factors have been implemented in the hypoglycemic presentation, and removal of the tumor leads to normoglycemia. ${ }^{8}$

Conclusion. Our case is typical of the Doege-Potter syndrome and is an example of a rare phenomenon that is infrequently described. It illustrates the size the tumor can reach before becoming symptomatic, our case being among the largest of those that have been reported. The case further demonstrates how difficult removal of such a sizeable mass from the hemithorax can be. We would recommend the combined approach of thoracotomy and sternotomy to allow en masse removal of a potentially malignant tumor of such dimensions. Our patient made an uneventful recovery without increased morbidity caused by the extended incision.

\section{REFERENCES}

1. Klemperer P, Rabin CB. Primary neoplasms of the pleura: a report of five cases. Arch Pathol 1931;11:385-412.
2. Taccagni G, Sambade C, Nesland J, Terreni MR, SobrinhoSimones M. Solitary fibrous tumour of the thyroid: clinicopathological, immunohistochemical and ultrastructural study of three cases. Virchows Arch A Pathol Anat Histopathol 1993;422:491-7.

3. Safneck JR, Alguacil-Garcia A, Dort JC, Phillips SM. Solitary fibrous tumor: a report of two new locations in the upper respiratory tract. J Laryngol Otol 1993;107:252-6.

4. Stringfellow HF, Khan IA, Sissions MC. Solitary fibrous tumor of the nasal cavity: report of a case. J Laryngol Otol 1996;110: 468-70.

5. Briselli M, Mark EJ, Dickersin GR. Solitary fibrous tumor of the pleura: eight new cases and review of 360 cases in the literature. Cancer 1981;47:2678-89.

6. Flint A, Weiss SW. CD-34 and keratin expression distinguishes solitary fibrous tumor of the pleura from desmoplastic mesothelioma. Hum Pathol 1995;26:428-31.

7. Doege KW. Fibrosarcoma of the mediastinum. Ann Surg 1930; 92:955-61.

8. Moat NE, Pawade A, Lewis BC, Shore G, Lamb RK, Monro JJ. Spontaneous hypoglycaemia and pleural fibroma: role of insulin like growth factors. Thorax 1991;46:932-33. 